\title{
An Unexpected Role of Cholesterol Sulfotransferase and its Regulation in Sensitizing Mice to Acetaminophen-Induced Liver Injury
}

\author{
Yunqi An, Pengcheng Wang, Pengfei Xu, Hung-Chun Tung, Yang Xie, Levent Kirisci, \\ Meishu Xu, Songrong Ren, Xin Tian, Xiaochao Ma, and Wen Xie \\ Center for Pharmacogenetics and Department of Pharmaceutical Sciences, (Y.A., P.W., P.X., H-C.T., Y.X., L.K., M.X., S.R., X.T., \\ X.M., W.X.) and Department of Pharmacology and Chemical Biology (W.X.), University of Pittsburgh, Pittsburgh, Pennsylvania; \\ and Department of Pharmacy, The First Affiliated Hospital of Zhengzhou University, Zhengzhou, China (X.T.)
}

Received October 5, 2018; accepted March 17, 2019

\section{ABSTRACT}

Overdose of acetaminophen (APAP) is the leading cause of acute liver failure (ALF) in the United States. The sulfotransferasemediated sulfation of APAP is widely believed to be a protective mechanism to attenuate the hepatotoxicity of APAP. The cholesterol sulfotransferase SULT2B1b is best known for its activity in catalyzing the sulfoconjugation of cholesterol to synthesize cholesterol sulfate. SULT2B1b can be transcriptionally and positively regulated by the hepatic nuclear factor $4 \alpha$ (HNF $4 \alpha)$. In this study, we uncovered an unexpected role for SULT2B1b in
APAP toxicity. Hepatic overexpression of SULT2B1b sensitized mice to APAP-induced liver injury, whereas ablation of the Sult2B1b gene in mice conferred resistance to the APAP hepatotoxicity. Consistent with the notion that Sult2B1b is a transcriptional target of $\mathrm{HNF} 4 \alpha$, overexpression of HNF $4 \alpha$ sensitized mice or primary hepatocytes to APAP-induced hepatotoxicity in a Sult2B1bdependent manner. We conclude that the HNF $4 \alpha$-SULT2B1b axis has a unique role in APAP-induced acute liver injury, and SULT2B1b induction might be a risk factor for APAP hepatotoxicity.

\section{Introduction}

Acetaminophen (APAP), or Tylenol, is a commonly used drug to reduce fever and relieve pain; however, APAP overdoses result in approximately 500 deaths each year in the United States (Lee, 2008). APAP overdose causes most of the acute liver failures (ALF), especially in the United States (Furuta et al., 2016). At therapeutic doses, about 3\% of APAP is excreted as the parent drug via the urine. More than $90 \%$ of APAP can be rapidly metabolized into the nontoxic compounds by phase 2 conjugating enzymes in the liver, twothirds through glucuronidation by UDP-glucuronosyltransferases (UGTs) and one third through sulfation by sulfotransferases (SULTs). From 5\% to 9\% of APAP is bioactivated to a highly reactive toxic metabolite, $\mathrm{N}$-acetyl-p-benzoquinone imine (NAPQI), by phase 1 cytochrome P450 enzymes (P450s), especially the CYP2E1 (Potter et al., 1973; Dahlin et al., 1984; Mannery et al., 2010). NAPQI has a short half-life because it can be rapidly eliminated by conjugation with glutathione (GSH) before being excreted via the urine as mercapturic acid

The work was supported in part by the National Institutes of Health [Grants DK083952, ES023438, DK117370]. W.X. was supported in part by the Joseph Koslow endowed Chair Professorship from the University of Pittsburgh School of Pharmacy.

https://doi.org/10.1124/mol.118.114819. and cysteine conjugates (Du et al., 2016). When APAP overdose occurs, the glucuronidation and sulfation pathways are saturated, so more APAP is metabolized by P450s to NAPQI. Excessive NAPQI depletes intracellular GSH. Accumulated NAPQI binds to other cellular proteins, leading to mitochondrial oxidative stress, nuclear DNA fragmentation, and hepatocyte death (Beger et al., 2015; Du et al., 2016). The oral formulation of $\mathrm{N}$-acetylcysteine (NAC) is an antidote approved for treating the overdose of APAP. NAC is nearly fully hepatoprotective when it is administered to patients within 8 hours after an acute APAP overdose (Wolf et al., 2007; Yoon et al., 2016); however, NAC has a narrow therapeutic window (Nam et al., 2017). There are no effective treatment options for severe ALF except liver transplantation. Thus, new therapeutics that target APAP overdose are urgently needed.

The human SULT2 family, also called the hydroxysteroidSULT family, comprises two genes, known as SULT2A1 and SULT2B1. They catalyze the sulfo-conjugation of many compounds and differ in tissue distribution and substrate specificity (Falany and Rohn-Glowacki, 2013). Because of the length of transcripts, SULT2B1 is divided into two isoforms, SULT2B1a and SULT2B1b. At the transcript level, the expression of SULT2B1a and SULT2B1b is very similar, but only the SULT2B1b protein can be detected in humans

ABBREVIATIONS: ALF, acute liver failure; ALT, alanine aminotransferase; APAP, acetaminophen; APAP-CYS, APAP-cysteine; AST, aspartate aminotransferase; CAR, constitutive androstane receptor; GSH, glutathione; HNF4 $\alpha$, hepatic nuclear factor $4 \alpha$; IL, interleukin; KO, knockout; LXR, liver X receptor; NAC, N-acetylcysteine; NAPQI, N-acetyl-p-benzoquinone-imine; P450, cytochrome P450; pcna, proliderating cell nuclear antigen; PCR, polymerase chain reaction; PXR, pregnane $X$ receptor; SULT, sulfotransferase; SULT2B1b, cholesterol sulfotransferase; TG, transgenic; TUNEL, in situ DNA nick end labeling; UGT, UDP-glucuronosyltransferase. 
(Falany et al., 2006). SULT2B1b appears to be the major functional SULT2B1 isoform. SULT2B1b is specific for the sulfation of 3-hydroxysteroids (Falany and Rohn-Glowacki, 2013). Subsequent studies have reported crucial roles of SULT2B1b in regulating liver functions and impacting the pathogenesis of diseases. SULT2B1b is induced by liver regeneration in a mouse model of partial hepatectomy (Lo Sasso et al., 2010). SULT2B1b increases the proliferation of liver cancer cells, which may have contributed to the progression of hepatocellular carcinoma (Yang et al., 2013). Oxysterols, the endogenous ligands for the liver $\mathrm{X}$ receptor (LXR), are substrates of SULT2B1b (Bensinger et al., 2008; Bai et al., 2011). Upregulation of SULT2B1b inhibited lipogenesis by sulfonating and deactivating the LXR-activating oxysterols (Bai et al., 2011) and aggravated 3,5-diethoxycarbonyl-1,4dihydrocollidine-induced liver damage by suppressing oxysterolinduced LXR activation (Wang et al., 2017). SULT2B1b has been reported to be regulated by several nuclear receptors, such as chimeric antigen receptor (Dong et al., 2009), vitamin D receptor (Seo et al., 2013), and peroxisome proliferator activated receptors (Jiang et al., 2005).

We recently reported that SULT2B1b can inhibit hepatic gluconeogenesis by suppressing the gluconeogenic activity of hepatocyte nuclear factor $4 \alpha(\mathrm{HNF} 4 \alpha)$ (Shi et al., 2014). HNF $4 \alpha$ promotes gluconeogenesis by upregulating PEPCK and G6Pase gene expression (Rhee et al., 2003). Mechanistically, SULT2B1b and its enzymatic product cholesterol sulfate suppress gluconeogenesis by inhibiting acetyl-CoA synthetase gene expression, leading to reduced acetylation and nuclear exclusion of HNF4 $\alpha$ (Shi et al., 2014). More recently, we reported that the SULT2B1b itself is a transcriptional target gene of $\mathrm{HNF} 4 \alpha$ (Bi et al., 2018). The establishment of SULT2B1b as a HNF4 $\alpha$ target gene called up our hypothesis that the induction of SULT2B1b by $\mathrm{HNF} 4 \alpha$ involves in a negative feedback to inhibit the gluconeogenic activity of HNF $4 \alpha$ (Bi et al., 2018). Although several SULT isoforms and their regulations have been implicated in APAP toxicity (Saini et al., 2011), it is unknown whether SULT2B1b plays a role in APAP hepatotoxicity and, if so, whether HNF $4 \alpha$ can also impact the hepatotoxicity of APAP by its positive regulation of SULT2B1b.

In this study, we demonstrated that overexpression of hepatic SULT2B1b sensitized mice to APAP-induced liver damage, whereas ablation of Sult2B1b attenuated mice from APAP toxicity. A forced expression of $\mathrm{Hnf} 4 \alpha$ aggravates APAP hepatotoxicity in a Sult2B1b-dependent manner. Our results pointed to SULT2B1b induction as a potential risk factor for APAP-induced acute liver damage.

\section{Materials and Methods}

Animals. The Sult2B1b knockout mice (strain no. 018773) in C57BL/6J-129/SvJ mixed background were purchased from the Jackson Laboratory (Bar Harbor, ME), and wild-type (WT) mice of the same mixed genetic background were used as controls. The liverspecific fatty acid binding protein-SULT2B1b transgenic mice in the C57BL/6J background were created and characterized as previously described (Shi et al., 2014), and WT C57BL/6J mice were used as controls. All mice used in this study were 6- to 8-week-old female mice. The use of animals in this study complied with all relevant federal guidelines and institutional policies.

Induction of APAP-Induced Liver Injury. APAP was dissolved in $0.5 \%$ methyl cellulose solution. Before receiving a single dose of $200 \mathrm{mg} / \mathrm{kg}$ APAP by gavage, all the mice were fasted for 16 hours. Food was given back to mice 3 hours after the APAP treatment. The mice were sacrificed 24 hours after APAP treatment (Saini et al., 2011). Liver tissues and serum samples were collected for biochemical analysis and histology. All chemicals mentioned in this study were bought from Sigma (St. Louis, MO).

Histology. For H\&E staining, liver samples were fixed in $10 \%$ paraformaldehyde, embedded in paraffin, sectioned at $4 \mu \mathrm{m}$, and stained. For immunohistochemistry analysis, standard immunohistochemical procedures were performed using a Ki67 antibody purchased from Abcam (Cambridge, MA). APO-BRDU (in situ DNA nick end labeling, TUNEL) apoptosis kit from Novus (Littleton, CO) was used to examine dying cells with exposed or fragmented DNA ends.

Serum and Liver Tissue Chemistry. ALT and AST analysis in the serum (Lu et al., 2015) and primary hepatocytes (Miyakawa et al., 2015) was performed using commercial assay kits from Stanbio Laboratory (Boerne, TX). The ratio of ALT in the medium of primary hepatocytes to ALT in the lysates of primary hepatocytes was calculated as the percentage of ALT release. The concentrations of GSH and GSSG in the liver tissue samples were measured by Assay Kit from BioAssay Systems (Hayward, CA).

Real-Time Polymerase Chain Reaction. Total RNA in the liver was isolated by the TRIzol reagent from Thermo Fisher Scientific (Pittsburgh, PA). Reverse transcription was conducted with random hexamer primers and Superscript RT III enzyme from Invitrogen. The ABI 7300 Real-Time PCR system was used to perform SYBR Greenbased real-time polymerase chain reaction (PCR). The quantity of mRNA measured was normalized to the cyclophilin gene expression.

Western Blot Analysis. Western blotting was conducted as described previously (Shi et al., 2014). The primary antibodies for Hnf4 $\alpha$ (catalog no. MA1-199) and Sult2B1b (sc-67103) were ordered from Thermo Fisher Scientific and Santa Cruz (Santa Cruz, CA), respectively.

Isolation, Culture, and Adenoviral Infection of Primary Hepatocytes from Mice. As described previously (Jiang et al., 2014), primary hepatocytes were extracted from 8- to 10-week-old mice. Briefly, the mice liver was first perfused with Hanks' buffered salt solution containing $0.1 \mathrm{M}$ HEPES and $0.5 \mathrm{mM}$ EGTA at the speed of $5 \mathrm{ml} / \mathrm{min}$ for $5-10$ minutes and then perfused with L-15 medium containing $20 \mathrm{mg} / \mathrm{ml}$ liberase, $1.8 \mathrm{mM} \mathrm{CaCl}_{2}$, and $0.1 \mathrm{M}$ HEPES from Roche (Indianapolis, IN). When the perfusion was completed, the dissociated hepatocytes were filtered through $50-\mathrm{mm}$ tissue cell strainer and centrifuged at $500 \mathrm{rpm}$ for 3 minutes at $4^{\circ} \mathrm{C}$ to collect. Hepatocytes were seeded onto six-well plates coated with type 1 collagen in William E medium containing $5 \%$ fetal bovine serum. After 2 hours, the medium was replaced by HepatoZYME-SFM medium (GIBCO, Grand Island, NY). To overexpress Hnf $4 \alpha$, the primary hepatocytes were infected with adenovirus expressing $\mathrm{Hnf} 4 \alpha$ (Ad-Hnf $4 \alpha$ ), or the control virus (Ad-Ctrl). Both viruses were given by Dr. Yanqiao Zhang from the Northeast Ohio Medical University (Yin et al., 2011).

APAP Metabolic Analysis. APAP metabolic analysis was performed as described (Cheng et al., 2009). In brief, $100 \mathrm{mg}$ of liver tissue samples was homogenized in $500 \mu \mathrm{l}$ of water. Two hundred microliters of acetonitrile/methanol $(1: 1, \mathrm{v} / \mathrm{v})$ was added to $100 \mu \mathrm{l}$ of each homogenate. The mixture was vortexed and centrifuged $(15,000 \mathrm{~g}$ for 10 minutes), and the supernatant was transferred to a new $1.5-\mathrm{ml}$ Eppendorf vial for a second centrifugation $(15,000 \mathrm{~g}$ for 10 minutes). Twenty microliters of the serum samples was added by $80 \mu \mathrm{l}$ of methanol. The prepared mixture was vortexed and centrifuged at $15,000 \mathrm{~g}$ for 10 minutes. Each supernatant was transferred to an autosampler vial and analyzed by ultraperformance liquid chromatography coupled with time-of-flight mass spectrometry (MS) from Waters Corporation (Milford, MA). Metabolites were separated by an Acquity UPLC BEH C18 column $(2.1 \times 50 \mathrm{~mm}, 1.7 \mu \mathrm{m}$; Waters $)$ using a gradient ranging from $5 \%$ to $95 \%$ aqueous acetonitrile containing $0.1 \%$ formic acid over a 6 -minute run. Time-of-flight MS was operated in positive mode with electrospray ionization, and the MS data were 
acquired in centroid format (50-1000 Da). The capillary and cone voltages were set as $0.8 \mathrm{kV}$ and $40 \mathrm{~V}$, respectively. The source temperature was set at $150^{\circ} \mathrm{C}$. The desolvation gas (800 liters/h) was set at $500^{\circ} \mathrm{C}$. APAP and its major metabolites (APAP-sulfate, APAPglucuronide, APAP-GSH, and APAP-Cys) were identified by highresolution accurate mass and tandem MS fragmental analysis. The peak areas were quantified to represent the signal intensities. All data were acquired using Masslynx V4.1 software and quantified using Quanlynx V4.1 (Waters Corp.).

Statistical Analysis. All the data are expressed as means \pm S.E.M. Comparisons of two groups were evaluated by the unpaired two-tailed Student's $t$ test, and comparisons of three or more groups were performed by one-way multivariant analysis of variance, which was carried out by SPSS version 24.0. to understand whether there were differences between the genotype on the two dependent variables, AST and ALT. The criterion for statistical significance was a $P$ value of less than 0.05 unless otherwise indicated. Some results had Bonferroni correction to control the type 1 errors and accepted a $P$ value of less than 0.005 (for Figs. 1 and 2, in which 10 comparisons were tested in the same samples), 0.017 (for Fig. 3, in which three comparisons were tested in the same samples; not significant in
Fig. 4), 0.003 (for Fig. 5, A-C, in which 15 comparisons were tested in the same samples), 0.025 (for Fig. $5 \mathrm{E}$, in which two comparisons were tested in the same samples), or 0.01 (for Fig. $5 \mathrm{~F}$, in which five comparisons were tested in the same samples).

\section{Results}

Transgenic Overexpression of SULT2B1b in the Liver Aggravates APAP-Induced Liver Injury. We previously reported the creation and characterization of transgenic (TG) mice that overexpress the cholesterol sulfotransferase (SULT2B1b) in the liver under the rat fatty acid binding protein gene promoter (Shi et al., 2014). To investigate whether SULT2B1b plays a role in APAP hepatotoxicity, we treated the TG mice with a single dose of $\operatorname{APAP}(200 \mathrm{mg} / \mathrm{kg}$ by gavage $)$ after a 16-hour fasting (Saini et al., 2011). Twenty-four hours after treatment, liver tissue and serum samples were harvested for analysis. H\&E staining revealed that the livers of the TG mice displayed more severe damage compared with the WT mice.
A

A
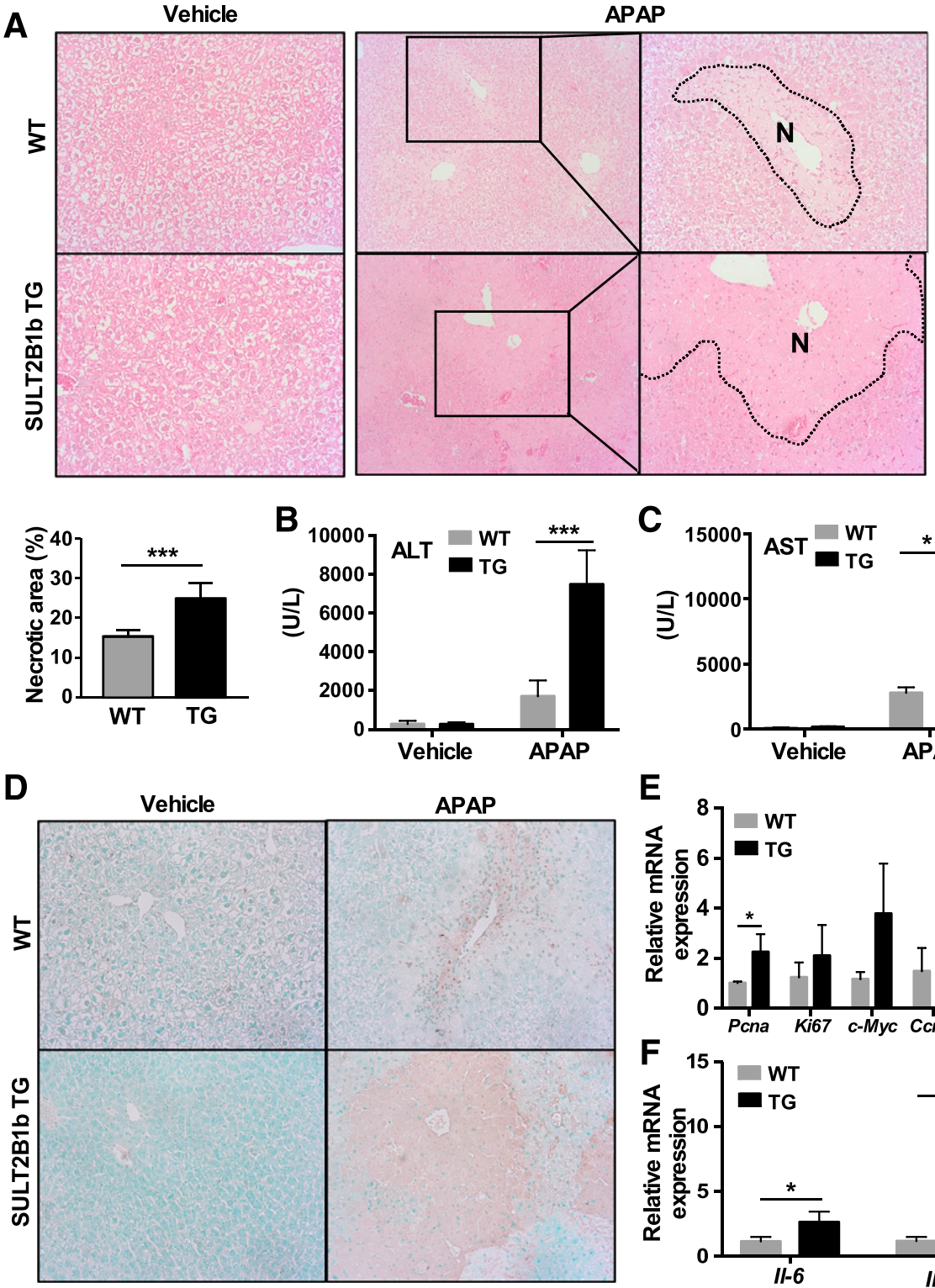

C

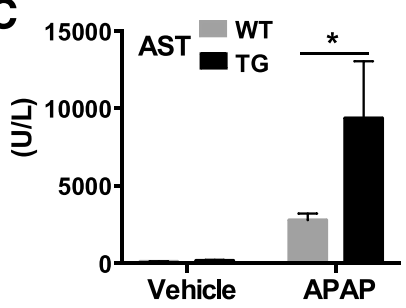

E
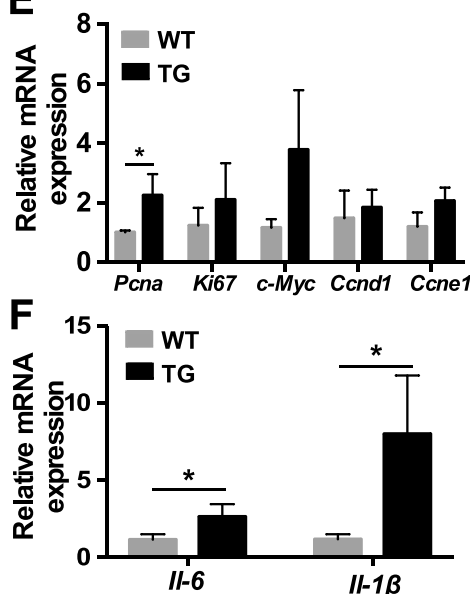

Fig. 1. Transgenic overexpression of SULT2B1b in the liver aggravates APAPinduced liver injury. (A) Representative H\&E staining on liver paraffin sections from WT and SULT2B1b TG mice treated with vehicle (left, original magnification, $100 \times$ ) or APAP (middle, original magnification, $100 \times$ ) for 24 hours. Shown on the right is anenlarged view of the boxed region in the middle panels highlighting the APAP-induced centrilobular necrosis. Shown on the bottom left is the quantification of the necrotic areas. $n=$ 5 per group. (B and C) Serum levels of ALT (B) and AST (C) in WT and TG mice treated with vehicle or APAP. (D) Representative images of TUNEL staining in liver sections from WT and TG mice 24 hours after vehicle or APAP treatment (original magnification, $200 \times$ ). (E) Relative hepatic mRNA expression of Pcna, Ki67, C-myc, Ccnd1, and Ccne1. (F) Relative hepatic mRNA expression of $I l-6$ and $I l-1 \beta$. Data are expressed as mean \pm S.E.M. $* P<0.05 ; * * P<0.01 ; * * * P<0.005$ (considered statistically significant) upon Bonferroni correction, compared with WT within the same drug treatment (B and $\mathrm{C}$ ) or the same gene ( $\mathrm{E}$ and $\mathrm{F}$ ) as labeled. 
A

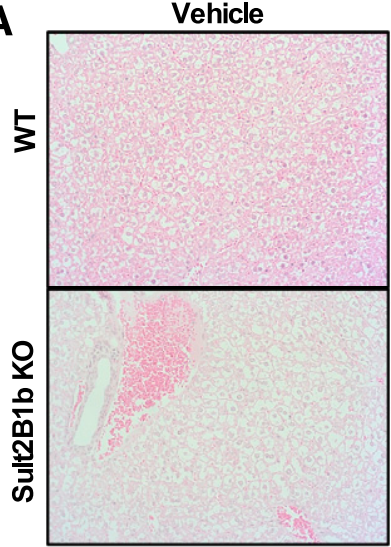

B

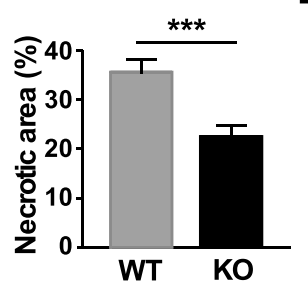

D

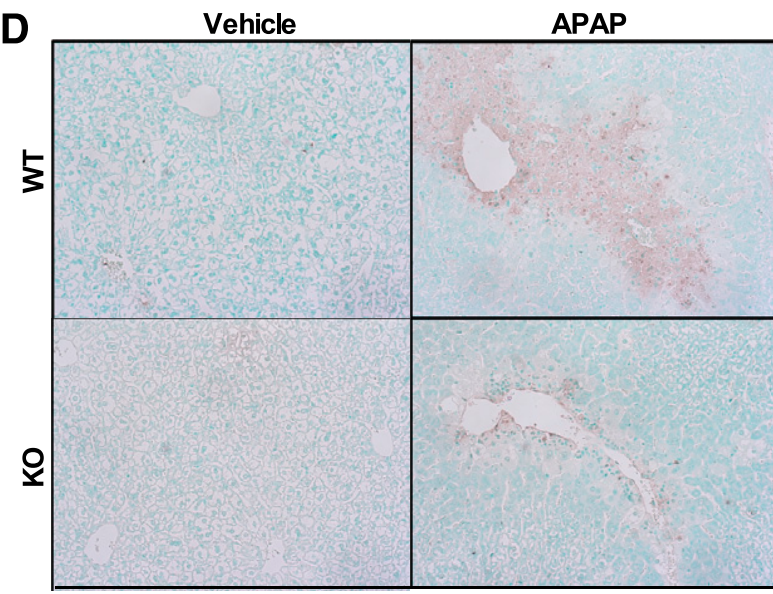

$\mathbf{F}$

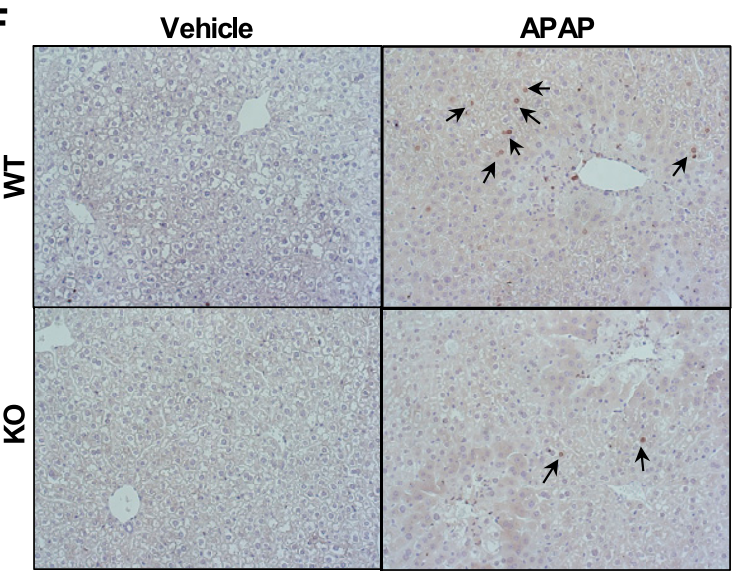

APAP

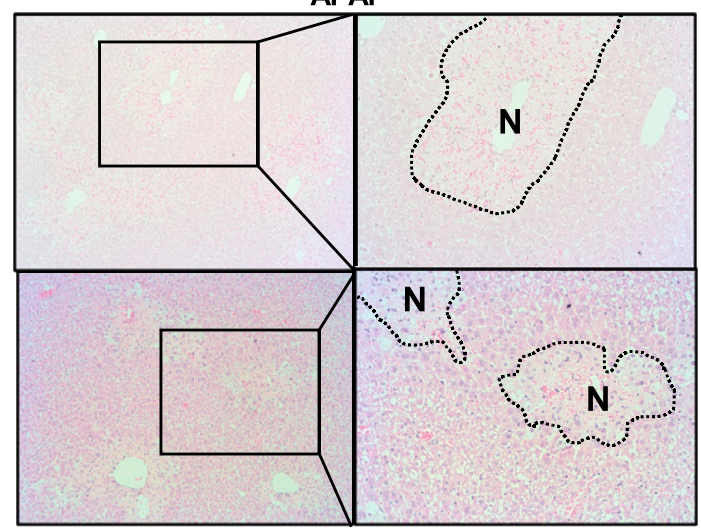

C

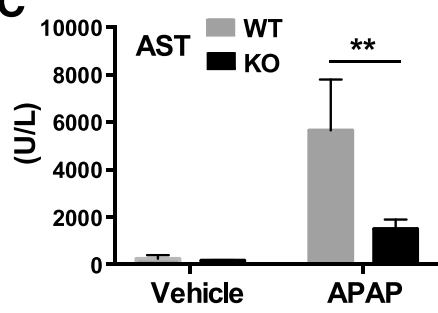

E
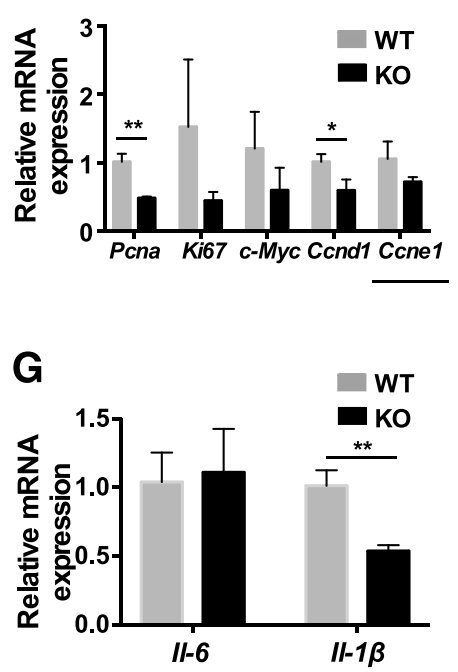

Fig. 2. Ablation of Sult2B1b protects mice from APAP-induced liver injury. (A) Representative $H \& E$ staining on liver paraffin sections from WT and Sult2B1b KO mice treated with vehicle (left, original magnification, $100 \times$ ) or APAP (middle, original magnification, $100 \times$ ) for 24 hours. Shown on the right is an enlarged view of the boxed region in the middle panels highlighting the APAP-induced centrilobular necrosis. Shown on the bottom left is the quantification of the necrotic areas. $n=3$ for WTvehicle and KO-vehicle, $n=8$ for WT-APAP and KO-APAP. (B and C) Serum levels of ALT (B) and AST (C) in WT and KO mice treated with vehicle or APAP. (D) Representative images of TUNEL staining in liver sections from WT and KO mice 24 hours after the vehicle or APAP treatment (original magnification, $200 \times$ ). (E) Relative hepatic mRNA expression of Pcna Ki67, C-myc, Ccnd1, and Ccne1. (F) The expression of Ki67 was detected by immunohistochemistry; arrows indicate positive stainings. (G) Relative hepatic mRNA expression of $\mathrm{Il}-6$ and $\mathrm{Il}-1 \beta$. Data are expressed as mean \pm S.E.M. ${ }^{*} P<0.05$; $* * P<0.01 ; * * * P<0.005$ (considered statistically significant) upon Bonferroni correction compared with WT within the same drug treatment (B and $\mathrm{C}$ ) or the same gene (E and G) as labeled.
The liver histology of the vehicle-treated TG mice appeared normal. Upon the APAP treatment, the TG mice showed quantifiably more extensive necrotic liver damage than their WT counterparts (Fig. 1A). In addition, APAP-treated TG mice had elevated serum levels of alanine aminotransferase (ALT)
(Fig. 1B) and aspartate aminotransferase (AST) (Fig. 1C) activity compared with APAP-treated WT mice. In APAPinduced liver injury, it is believed that overdose of APAP is metabolized by $\mathrm{P} 450$ s to reactive intermediate NAPQI, which depletes GSH, binds to cellular proteins, and induces nuclear 

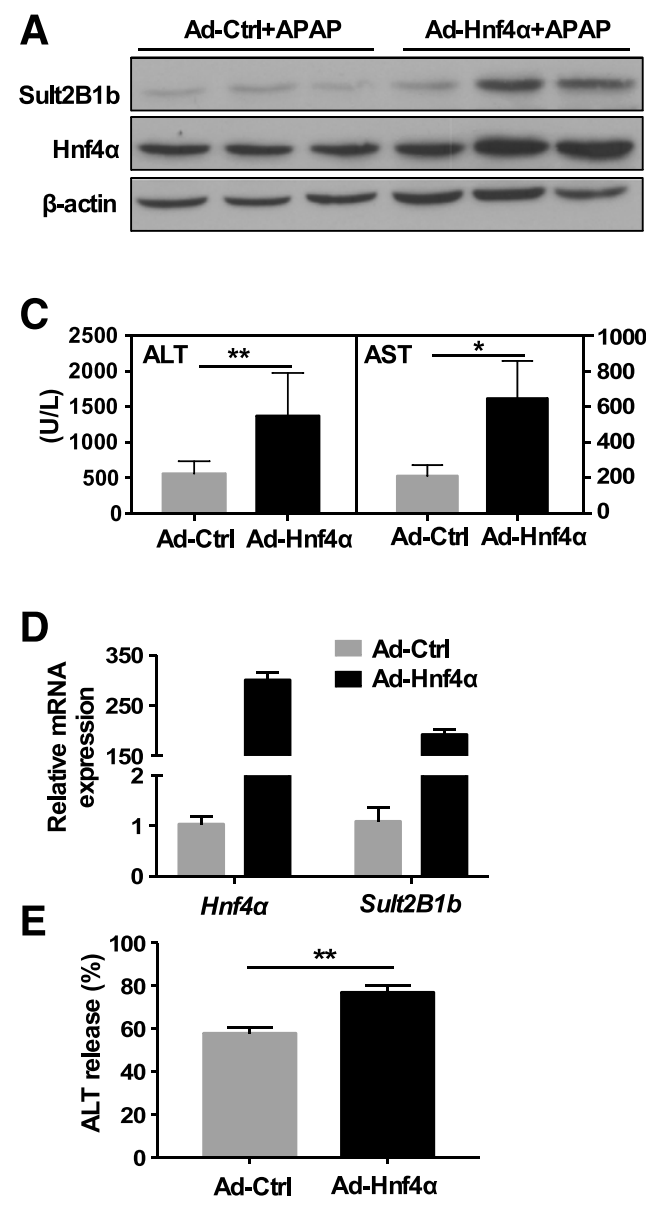

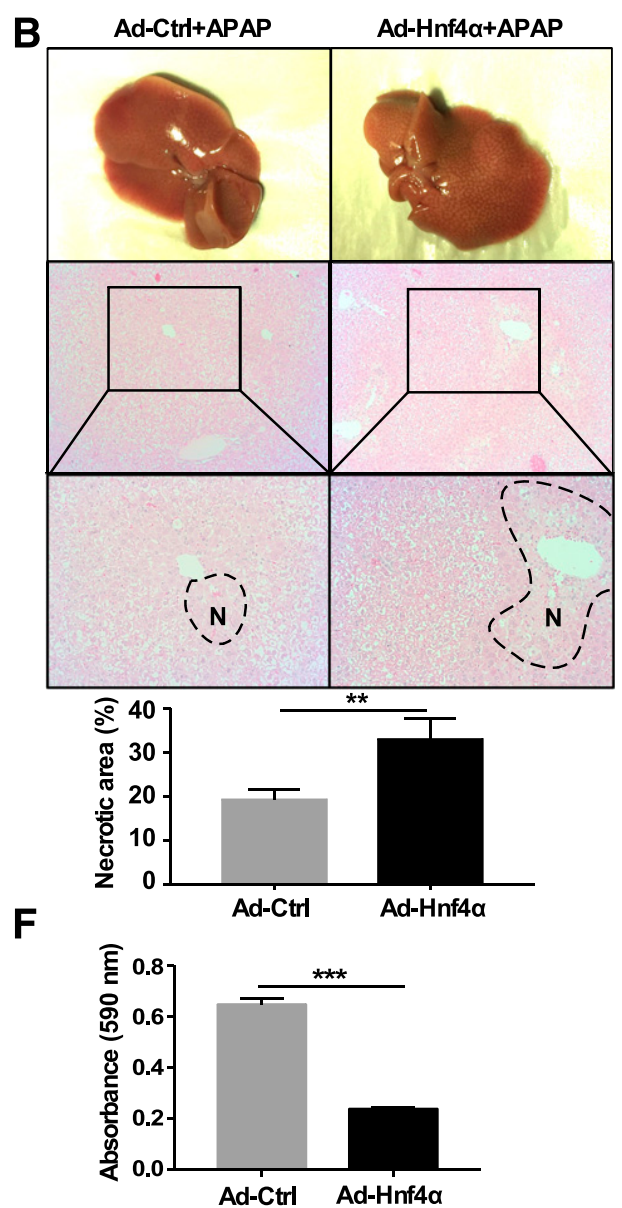

Fig. 3. Overexpression of $\mathrm{Hnf} 4 \alpha$ induces Sult2B1b and sensitizes mice or primary hepatocytes to APAP-induced injury. (A) Hepatic expression of Sult2B1b and Hnf $4 \alpha$ in WT mice infected with Ad-Ctrl or Ad-Hnf4 and treated with APAP was measured by Western blot analysis. $n=$ 3 per group. (B) Representative gross appearance (top) and H\&E staining on liver paraffin sections of Ad-Ctrl+APAP and Ad-Hnf4+APAP WT mice (middle, original magnification, $100 \times$ ) and an enlarged view of the boxed region in the middle panels highlighting the APAP-induced centrilobular necrosis (bottom). Shown below is the quantification of the necrotic areas. $n=3$ for Ad-Ctrl, $n=5$ for Ad-Hnf4. (C) Serum levels of ALT and AST in WT mice infected with Ad-Ctrl or Ad-Hnf4 $\alpha$. (D) The mRNA expression of $\mathrm{Hnf} 4 \alpha$ and Sult2B1b in WT primary hepatocytes infected with Ad-Ctrl or Ad-Hnf4 $\alpha$. (E) The percentage of ALT release from WT primary hepatocytes infected with Ad-Ctrl or Ad-Hnf4 $\alpha$ and treated with $5 \mathrm{mM}$ APAP for 24 hours. (F) MTT assay on primary hepatocytes described in (E). Data are expressed as mean \pm S.E.M. $* P<0.05 ; * * P<0.017$ $* * * P<0.001$ (considered statistically significant) upon Bonferroni correction, all compared with Ad-Ctrl as labeled.
DNA fragmentation, leading to necrotic hepatocyte death (Hinson et al., 2010). So, we assessed nuclear DNA fragmentation by TUNEL staining. The TUNEL staining in APAPtreated TG mice was increased (Fig. 1D), consistent with the increased liver damage in this genotype. It is known that liver regeneration is important for survival after APAP overdose (Bhushan et al., 2014) because hepatic cell necrosis eventually induces liver regeneration (Guicciardi et al., 2013). Usually, more necrosis is followed by more cell proliferation. Therefore, we evaluated the expression of cell proliferation and cell-cyclerelated genes in the liver by real-time PCR. The expression of proliferating cell nuclear antigen (Pcna) of TG mice was significantly higher than the WT mice (Fig. 1E), consistent with the notion that TG mice had more liver damage than WT mice. Furthermore, the hepatic expression of inflammatory cytokine genes interleukin-6 (Il-6) and interleukin-1 $\beta$ ( $I l-1 \beta)$ in TG mice was significantly higher than that in WT mice after the APAP treatment (Fig. 1F).

Ablation of Sult2B1b Protects Mice from APAPInduced Liver Damage. To assess whether Sult2B1b ablation affects APAP-induced liver injury, we treated Sult2B1b knockout (KO) mice with a single dose of $200 \mathrm{mg} / \mathrm{kg}$ APAP. The $\mathrm{KO}$ mice showed less liver damage compared with the WT mice. The WT mice showed remarkable necrotic liver damage at 24 hours post-APAP treatment, as expected. In contrast, the $\mathrm{KO}$ mice showed less liver damage as evidence by smaller necrotic area (Fig. 2A), decreased serum levels of ALT (Fig. 2B) and AST (Fig. 2C), and decreased TUNEL staining (Fig. 2D). The mRNA expression of Pcna and Ccnd 1 was significantly decreased in APAP-treated KO mice (Fig. 2E), consistent with an attenuated liver injury in this genotype. The decreased hepatocyte proliferation in APAP-treated KO mice was also supported by a decreased immunostaining of the hepatocyte proliferation marker Ki67 (Fig. 2F). Consistently and compared with the WT mice, mRNA expression of $I l-1 \beta$ was decreased in APAP-treated KO mice (Fig. 2G).

Overexpression of $\mathrm{Hnf4 \alpha}$ Induces Sult2B1b and Sensitizes Mice or Primary Hepatocytes to APAPInduced Injury. We recently reported that Sult2B1b is a transcriptional target of $\mathrm{Hnf} 4 \alpha$ (Bi et al., 2018). Having shown that overexpression of SULT2B1b sufficed to sensitize mice to APAP-induced liver injury, we wanted to determine whether upregulation of Sult2B1b by $\mathrm{HNF} 4 \alpha$ in the mouse liver will have a similar sensitizing effect on APAP-induced liver injury. In this experiment, WT mice were infected with adenovirus expressing $\mathrm{Hnf} 4 \alpha$ (Ad-Hnf4 $\alpha$ ) or the control virus (Ad-Ctrl) for 1 week before being treated with APAP. The overexpression Hnf $4 \alpha$ and expected induction of Sult2B1b were confirmed by Western blotting (Fig. 3A). Compared with Ad-Ctrl infected mice, the Ad-Hnf $4 \alpha$ infected mice displayed more severe liver injury as evidenced by the gross appearance and increased necrotic area (Fig. 3B) and increased serum levels of ALT and AST (Fig. 3C). The in vivo results were further supported by in vitro results using primary hepatocytes isolated from WT mice. In this experiment, primary hepatocytes infected with Ad-Ctrl or Ad-Hnf $4 \alpha$ were treated with $5 \mathrm{mM}$ APAP for 24 hours. Overexpression of $\mathrm{Hnf} 4 \alpha$ and induction of Sult2B1b were proved by real-time PCR (Fig. 3D). Compared with 
A

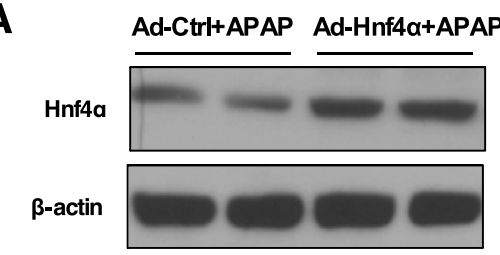

C

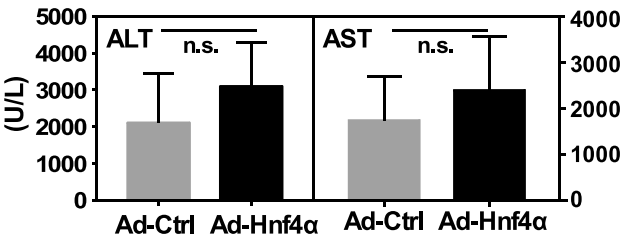

D

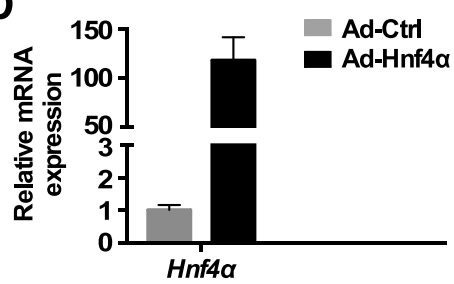

$\mathbf{F}$

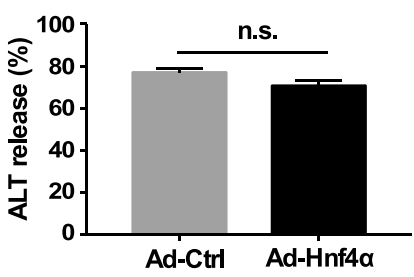

B

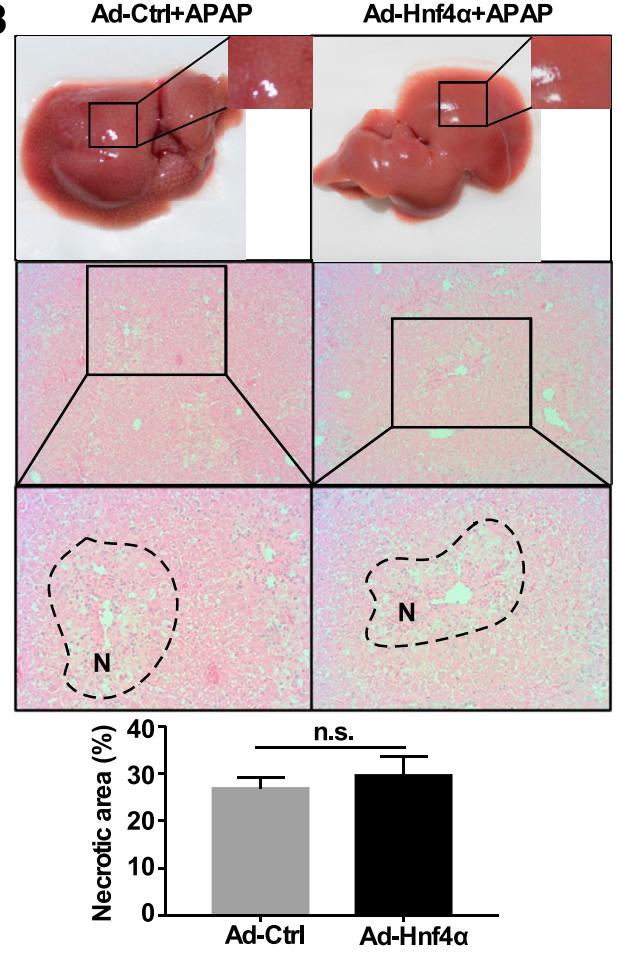

E

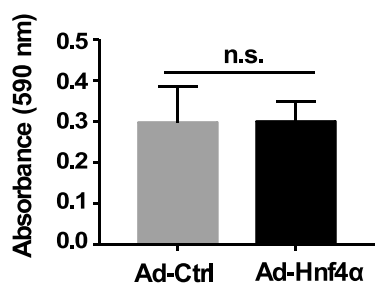

Fig. 4. The sensitizing effect of HNF $4 \alpha$ on APAP-induced injury is Sult2B1bdependent. (A) Hepatic expression of Sult2B1b in Sult2B1b KO mice infected with Ad-Ctrl or Ad-Hnf4 and treated with APAP was measured by Western blot analysis. $n=3$ per group. (B) Representative gross appearance (top) and $\mathrm{H} \& \mathrm{E}$ staining on liver paraffin sections of Ad-Ctrl + APAP and Ad-Hnf4 + APAP KO mice (middle, original magnification, $100 \times$ ), and enlarged view of boxed region in middle panels highlighting the APAPinduced centrilobular necrosis (bottom) Shown below is the quantification of the necrotic areas. $n=3$ for Ad-Ctrl, $n=5$ for Ad-Hnf4. (C) Serum levels of ALT and AST in KO mice infected with Ad-Ctrl or Ad-Hnf4 $\alpha$. (D) The mRNA expression of Hnf $4 \alpha$ in KO primary hepatocytes infected with Ad-Ctrl or Ad-Hnf4 $\alpha$. (E) The percentage of ALT release from KO primary hepatocytes infected with Ad-Ctrl or Ad-Hnf $4 \alpha$ and treated with $5 \mathrm{mM}$ APAP for 24 hours. (F) MTT assay on primary hepatocytes described in (E). Data are expressed as mean \pm S.E.M. n.s., statistically not significant, all compared with Ad-Ctrl as labeled.
Ad-Ctrl-infected hepatocytes, Ad-Hnf4 $\alpha$ infected hepatocytes showed an increased APAP-responsive release of ALT (Fig. $3 \mathrm{E})$ and a decreased cell viability as shown by the MTT assay (Fig. 3F).

Sensitizing Effect of HNF4 $\alpha$ on APAP-Induced Injury Is Sult2B1b- Dependent. To determine whether the sensitizing effect of $\mathrm{HNF} 4 \alpha$ on APAP-induced liver injury in vivo is Sult2B1b-dependent, we infected Sult2B1b KO mice with Ad-Ctrl or Ad-Hnf4 $\alpha$ before challenging them with APAP. The overexpression of $\mathrm{Hnf} 4 \alpha$ was verified by Western blotting (Fig. 4A). Upon APAP treatment, the injury between Ad-Ctrlinfected and Ad-Hnf $4 \alpha$-infected $\mathrm{KO}$ mice was not different because neither the gross appearance and necrotic area (Fig. 4B) nor the serum levels of ALT and AST (Fig. 4C) differed in these two groups of $\mathrm{KO}$ mice. The dependence of Sult2B1b in the sensitizing effect of $\mathrm{Hnf} 4 \alpha$ was also confirmed in primary hepatocytes isolated from the $\mathrm{KO}$ mice. The adenoviral overexpression of $\mathrm{Hnf} 4 \alpha$ in $\mathrm{KO}$ primary hepatocytes was confirmed by real-time PCR (Fig. 4D). The KO primary hepatocytes infected with Ad-Ctrl or Ad-Hnf $4 \alpha$ showed comparable release of ALT (Fig. 4E) and cell viability as shown by the MTT assay (Fig. 4F). These results suggested that the sensitizing effect of $\mathrm{HNF} 4 \alpha$ on APAP-induced injury is Sult2B1b-dependent both in vivo and in vitro.

Mechanism by which SULT2B1b Sensitizes Mice to APAP-Induced Liver Injury. To understand the mechanism by which SULT2B1b sensitizes mice to APAP-induced liver injury, we profiled the expression of genes known to play a role in APAP hepatotoxicity. Among the phase 1 and phase 2 enzymes, only the expression of Ugt1 was dramatically induced in APAP-treated TG mice, whereas the expressions of other APAP metabolizing enzymes showed no significant difference in either the TG or KO mice (Fig. 5A). The expression of a panel of nuclear receptors that are known to play a role in APAP hepatotoxicity, including constitutive androstane receptor (CAR) (Zhang et al., 2002), farnesoid X receptor (Lee et al., 2010), LXR $\alpha$ (Saini et al., 2011), pregnane X receptor (PXR) (Guo et al., 2004), and retinoid X receptor (Dai et al., 2005), was not affected by the SULT2B1b TG or $\mathrm{KO}$ either (Fig. 5B). We have previously reported that activation of $\mathrm{LXR} \alpha$ attenuated APAP-induced liver injury (Saini et al., 2011). We found the expressions of LXR, as well as its target genes, were not affected in APAP-treated TG or KO mice (Fig. 5C). The total hepatic GSH content in APAP-treated TG mice was remarkably decreased, whereas the ratio of GSSG/GSH was significantly increased in TG mice (Fig. 5D, top), consistent with the increased sensitivity to APAPinduced liver injury in this genotype; however, the total GSH level and the GSSG/GSH ratio between WT and KO mice were not different (Fig. 5D, bottom). Analysis of the APAP metabolites showed that the serum concentrations of APAP-sulfate and APAP-glucuronide were increased in TG mice 24 hours after the APAP treatment (Fig. 5E), consistent with the expression of the SULT transgene and induction of Ugt1 in this genotype. Interestingly, at 1 hour after APAP treatment, the transgene had little effect on the hepatic concentrations 

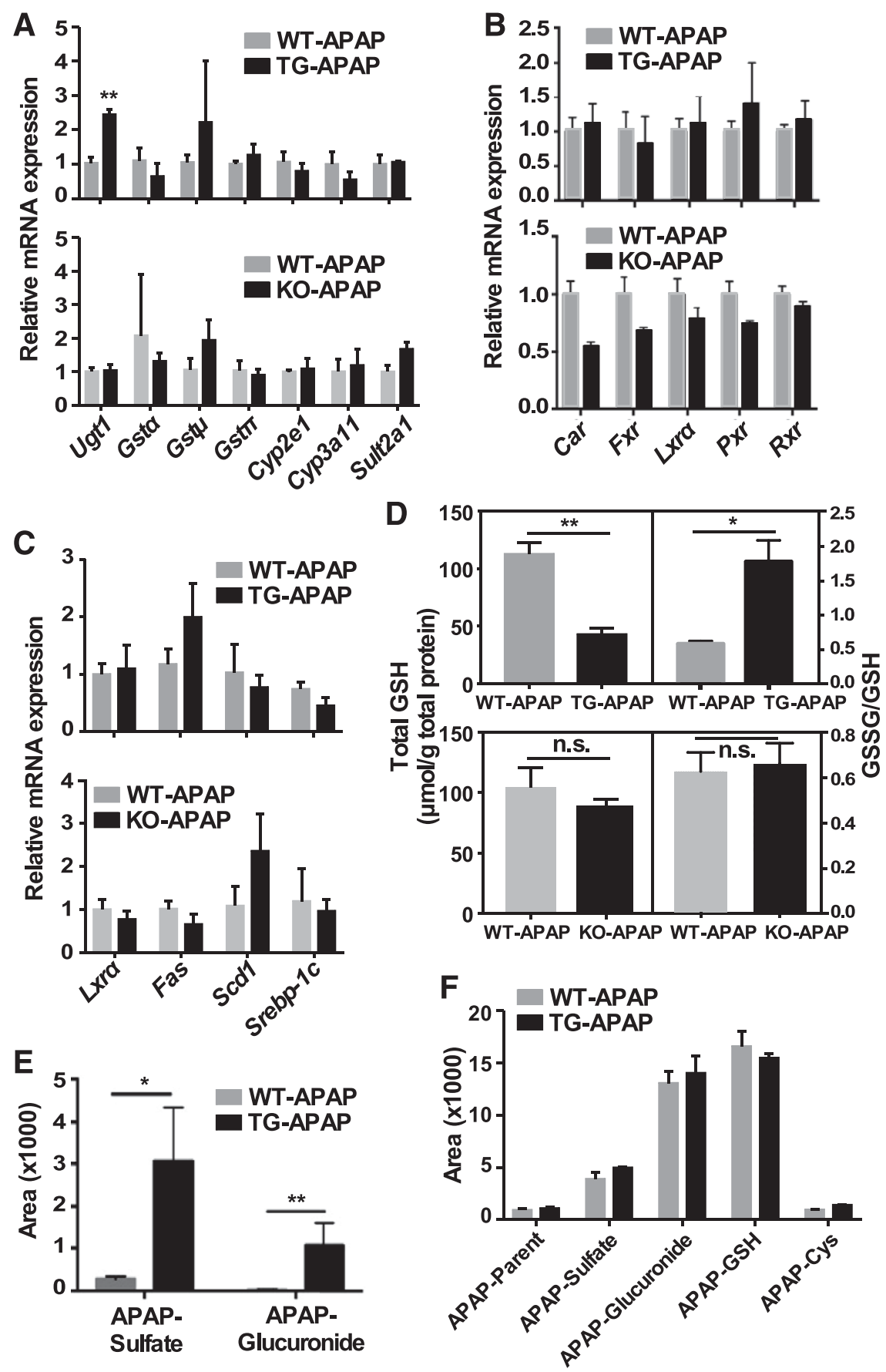

Fig. 5. Mechanism by which SULT2B1b sensitizes mice to APAP-induced liver injury. (A and B) Relative mRNA expression of a panel of phase 1 and phase 2 enzymes (A) and a panel of nuclear receptors (B) in the livers of WT, SULT2B1b TG and KO mice treated with APAP. $n=3$ per group. (C) Relative mRNA expression of $L X R \alpha$ and its target genes Fas, $S c d 1$, and $S r e b p-1 c$ in livers from WT, TG, and KO mice treated with APAP. $n=3$ per group. (D)Total liver GSH content and GSSG/GSH ratio in WT, TG, and $\mathrm{KO}$ mice treated with APAP. $n=3$ per group. (E) The contents of APAP-sulfate and APAP-glucuronide in the serum from WT and TG mice 24 hours after APAP treatment. $n=3$ per group. (F) The contents of parent APAP, APAP-sulfate, APAP-glucuronide, and APAP-Cys in the liver from WT and TG mice 1 hour after APAP treatment. $n=3$ per group. Data are expressed as mean \pm S.E.M. $* P<0.05 ; * * P<0.01$; $* * * P<0.003$ (considered statistically significant) upon Bonferroni correction for $(\mathrm{A}-\mathrm{C})$; $* P<0.05$; $* * P<0.01$ (considered statistically significant) for (D); ${ }^{*} P<0.05$; ${ }^{*} P<0.025$ (considered statistically significant) upon Bonferroni correction for (E); and $* P<0.01$ (considered statistically significant) upon Bonferroni correction for (F); n.s., statistically not significant, all compared with WT-APAP within the same gene $(\mathrm{A}-\mathrm{C})$ or the same parameter $(\mathrm{D}-\mathrm{F})$ as labeled. of parent APAP, APAP-sulfate, APAP-glucuronide, APAPGSH, or APAP-Cys (Fig. 5F).

\section{Discussion}

Overdose of APAP could eventually lead to ALF. Although the mechanisms of APAP-induced liver injury have been extensively studied, factors that can affect the progression of APAP-induced liver disease or improve the liver recovery are less investigated. Identification of the related pathogenic factors will provide novel therapeutic approaches for the treatment of APAP overdose.

SULT2B1b is a hydroxysteroid sulfotransferase that plays important roles in diverse cell types and tissues, such as suppressing lipogenesis (Ren and Ning, 2014) and gluconeogenesis (Shi et al., 2014; Bi et al., 2018) and promoting hepatocyte proliferation (Yang et al., 2013), affecting prostate and colorectal cancer cells (Vickman et al., 2016) and suppressing T-cell receptor signaling (Wang et al., 2016). In this study, we found a novel function of SULT2B1b in APAP-induced liver toxicity. Specifically, hepatic transgenic overexpression of SULT2B1b exacerbated APAP-induced acute liver injury, whereas ablation of the Sult2B1b gene in mice conferred protection to APAP hepatotoxicity. The sensitizing effect of SULT2B1b on APAP-induced liver injury is a surprise considering that sulfation of APAP has been established as an important mechanism for the detoxification of APAP. For example, we have previously reported 
the attenuation of APAP toxicity by the activation LXR $\alpha$ (Saini et al., 2011), which was associated with the induction of Sult2a1.

The mechanism by which SULT2B1b sensitizes mice to APAP toxicity remains to be clearly defined. APAP may not be a substrate of SULT2B1b in vitro, according to a published report (Yamamoto et al., 2015); however, we cannot exclude the possibility that APAP at the pharmacologic concentrations we used is a substrate of SULT2B1b in vivo, a notion supported by the increased serum concentration of APAPsulfate in TG mice 24 hours after the APAP treatment (Fig. 5E). Knowing that SULT2B1b suppresses LXR activity by sulfonating and deactivating the endogenous LXR agonists (Bensinger et al., 2008; Villablanca et al., 2010), and activation of LXR accelerates APAP clearance and attenuates APAP toxicity (Saini et al., 2011), we initially hypothesized that the sensitizing effect of SULT2B1b might be due to the inhibition of LXR; however, we found that the expression of LXR $\alpha$ and its primary target genes was not affected by the transgene (Fig. 5C). SULT2B1b transgene or ablation also had little effect on the expression of a panel of nuclear receptors and P450 enzymes that are known to impact APAP metabolism and toxicity, except that Ugt1 was upregulated in APAP-treated TG mice for a yet-to-be- defined reason (Fig. 5). The decreased GSH level in APAP-treated TG mice (Fig. 5D) suggested that overexpression of SULT2B1b may have compromised the replenishment of GSH via a yet-to-be-characterized mechanism. The hepatic concentrations of parent APAP, APAP-sulfate, APAP-glucuronide, and APAP-Cys 1 hour after APAP treatment showed no difference between the WT and TG mice. The serum concentrations of two nontoxic metabolites (APAPsulfate and APAP-glucuronide) were elevated 24 hours after APAP in the TG mice, but they were not affected in the $\mathrm{KO}$ mice. These results indicate that the effect of the SULT2B1b transgene on APAP metabolism might be time-dependent. A future detailed pharmacokinetic analysis is necessary to conclude the effect of SULT2B1b on APAP metabolism.

The sensitizing effect of $\mathrm{HNF} 4 \alpha$ is also intriguing. On one hand, the sensitization was predictable based on the positive regulation of Sult2B1b by $\mathrm{HNF} 4 \alpha$, as we have recently reported (Bi et al., 2018), and the sensitizing effect of HNF4 $\alpha$ was Sult2B1b-dependent (Fig. 4). On the other hand, aggravation of APAP-induced liver injury by $\mathrm{HNF} 4 \alpha$ was a surprise, considering the reported hepatoprotective effect of $\mathrm{HNF} 4 \alpha$ in liver injury induced by xenobiotic toxicants (Beggs et al., 2016), alcohol, and methionine/choline-deficient diet (Xu et al., 2016), as well as in the context of nonalcoholic steatogepatitis, ALF, and hepatocellular carcinoma (Shi et al., 2014; Vallianou et al., 2016; Baciu et al., 2017; Hang et al., 2017). To our knowledge, it is the first demonstration that HNF $4 \alpha$ aggravated APAP-induced liver injury through its transcriptional regulation of SULT2B1b. HNF4 $\alpha$ is reportedly critically involved in PXR- and CAR-mediated transcriptional activation of CYP3A (Tirona et al., 2003), and activation of PXR and CAR and induction of CYP3A are known to sensitize mice to APAP toxicity (Zhang et al., 2002; Guo et al., 2004; Cheng et al., 2009). Although the expression of PXR, CAR, and Cyp3a11 was not affected in APAP-treated TG or KO mice, we cannot exclude the possibility that PXR, CAR, and Cyp3a may play a role in mediating the sensitizing effect of HNF $4 \alpha$ on APAP toxicity. Female mice were used in this study. Our study focused on APAP toxicity in terms of its metabolism. It has been reported that APAP metabolism does not contribute to gender difference in the event of APAP overdose (Dai et al., 2006). Further studies are needed to determine whether the phenotype is sex-specific.

In summary, we have uncovered a novel function of SULT2B1b and its regulation by $\mathrm{HNF} 4 \alpha$ in APAP-induced acute liver injury. Our results suggest that SULT2B1b induction might be a risk factor for APAP hepatotoxicity. This notion is consistent with the report that nonalcoholic fatty liver disease sensitized rodents to APAP-induced liver injury (Michaut et al., 2014) because nonalcoholic fatty liver disease and its associated metabolic liver disease induce the hepatic expression of Sult2B1b (Shi et al., 2014).

\section{Authorship Contributions}

Participated in research design: An, W. Xie.

Conducted experiments: An, Wang, P. Xu, Tung, M. Xu, Ren, Tian. Performed data analysis: An, Wang, Y. Xie, Kirisci, W. Xie.

Wrote or contributed to the writing of the manuscript: An, Ma, W. Xie.

Note Added in Proof-NIH Grant number DK117370 was accidentally not included in the Fast Forward version published April 3, 2019. The funding footnote has now been corrected.

\section{References}

Baciu C, Pasini E, Angeli M, Schwenger K, Afrin J, Humar A, Fischer S, Patel K, Allard J, and Bhat M (2017) Systematic integrative analysis of gene expression identifies HNF4A as the central gene in pathogenesis of non-alcoholic steatohepatitis. PLoS One 12:e0189223.

Bai Q, Xu L, Kakiyama G, Runge-Morris MA, Hylemon PB, Yin L, Pandak WM, and Ren S (2011) Sulfation of 25-hydroxycholesterol by SULT2B1b decreases cellular lipids via the LXR/SREBP-1c signaling pathway in human aortic endothelial cells. Atherosclerosis 214:350-356.

Beger RD, Bhattacharyya S, Yang X, Gill PS, Schnackenberg LK, Sun J, and James LP (2015) Translational biomarkers of acetaminophen-induced acute liver injury. Arch Toxicol 89:1497-1522.

Beggs KM, McGreal SR, McCarthy A, Gunewardena S, Lampe JN, Lau C, and Apte U (2016) The role of hepatocyte nuclear factor 4-alpha in perfluorooctanoic acidand perfluorooctanesulfonic acid-induced hepatocellular dysfunction. Toxicol Appl Pharmacol 304:18-29.

Bensinger SJ, Bradley MN, Joseph SB, Zelcer N, Janssen EM, Hausner MA, Shih R, Parks JS, Edwards PA, Jamieson BD, et al. (2008) LXR signaling couples sterol metabolism to proliferation in the acquired immune response. Cell 134:97-111.

Bhushan B, Walesky C, Manley M, Gallagher T, Borude P, Edwards G, Monga SP, and Apte U (2014) Pro-regenerative signaling after acetaminophen-induced acute liver injury in mice identified using a novel incremental dose model. Am J Pathol 184:3013-3025.

Bi Y, Shi X, Zhu J, Guan X, Garbacz WG, Huang Y, Gao L, Yan J, Xu M, Ren S, et al. (2018) Regulation of cholesterol sulfotransferase SULT2B1b by hepatocyte nuclear factor $4 \alpha$ constitutes a negative feedback control of hepatic gluconeogenesis. $\mathrm{Mol}$ Cell Biol 38 DOI: 10.1128/MCB.00654-17.

Cheng J, Ma X, Krausz KW, Idle JR, and Gonzalez FJ (2009) Rifampicin-activated human pregnane $\mathrm{X}$ receptor and CYP3A4 induction enhance acetaminopheninduced toxicity. Drug Metab Dispos 37:1611-1621.

Dahlin DC, Miwa GT, Lu AY, and Nelson SD (1984) N-acetyl-p-benzoquinone imine: a cytochrome P-450-mediated oxidation product of acetaminophen. Proc Natl Acad Sci USA 81:1327-1331.

Dai G, Chou N, He L, Gyamfi MA, Mendy AJ, Slitt AL, Klaassen CD, and Wan YJ (2005) Retinoid X receptor alpha regulates the expression of glutathione $s$-transferase genes and modulates acetaminophen-glutathione conjugation in mouse liver. Mol Pharmacol 68:1590-1596.

Dai G, He L, Chou N, and Wan YJ (2006) Acetaminophen metabolism does not contribute to gender difference in its hepatotoxicity in mouse. Toxicol Sci 92:33-41.

Dong B, Saha PK, Huang W, Chen W, Abu-Elheiga LA, Wakil SJ, Stevens RD, Ilkayeva O, Newgard CB, Chan L, et al. (2009) Activation of nuclear receptor CAR ameliorates diabetes and fatty liver disease. Proc Natl Acad Sci USA 106: 18831-18836.

Du K, Ramachandran A, and Jaeschke H (2016) Oxidative stress during acetaminophen hepatotoxicity: sources, pathophysiological role and therapeutic potential. Redox Biol 10:148-156.

Falany CN, He D, Dumas N, Frost AR, and Falany JL (2006) Human cytosolic sulfotransferase 2B1: isoform expression, tissue specificity and subcellular localization. J Steroid Biochem Mol Biol 102:214-221.

Falany CN and Rohn-Glowacki KJ (2013) SULT2B1: unique properties and characteristics of a hydroxysteroid sulfotransferase family. Drug Metab Rev 45:388-400. Furuta K, Yoshida Y, Ogura S, Kurahashi T, Kizu T, Maeda S, Egawa M, Chatani N, Nishida K, Nakaoka Y, et al. (2016) Gab1 adaptor protein acts as a gatekeeper to balance hepatocyte death and proliferation during acetaminophen-induced liver injury in mice. Hepatology 63:1340-1355. 
Guicciardi ME, Malhi H, Mott JL, and Gores GJ (2013) Apoptosis and necrosis in the liver. Compr Physiol 3:977-1010.

Guo GL, Moffit JS, Nicol CJ, Ward JM, Aleksunes LA, Slitt AL, Kliewer SA, Manautou JE, and Gonzalez FJ (2004) Enhanced acetaminophen toxicity by activation of the pregnane X receptor. Toxicol Sci 82:374-380.

Hang H-L, Liu X-Y, Wang H-T, Xu N, Bian J-M, Zhang J-J, Xia L, and Xia Q (2017) Hepatocyte nuclear factor $4 \mathrm{~A}$ improves hepatic differentiation of immortalized adult human hepatocytes and improves liver function and survival. Exp Cell Res 360:81-93.

Hinson JA, Roberts DW, and James LP (2010) Mechanisms of acetaminopheninduced liver necrosis. Handb Exp Pharmacol 196:369-405.

Jiang M, He J, Kucera H, Gaikwad NW, Zhang B, Xu M, O’Doherty RM, Selcer KW, and Xie W (2014) Hepatic overexpression of steroid sulfatase ameliorates mouse models of obesity and type 2 diabetes through sex-specific mechanisms. J Biol Chem 289:8086-8097.

Jiang YJ, Kim P, Elias PM, and Feingold KR (2005) LXR and PPAR activators stimulate cholesterol sulfotransferase type 2 isoform $1 \mathrm{~b}$ in human keratinocytes. $J$ Lipid Res 46:2657-2666.

Lee FY, de Aguiar Vallim TQ, Chong HK, Zhang Y, Liu Y, Jones SA, Osborne TF, and Edwards PA (2010) Activation of the farnesoid X receptor provides protection against acetaminophen-induced hepatic toxicity. Mol Endocrinol 24:1626-1636.

Lee WM (2008) Acetaminophen-related acute liver failure in the United States. Hepatol Res 38 (Suppl 1):S3-S8.

Lo Sasso G, Celli N, Caboni M, Murzilli S, Salvatore L, Morgano A, Vacca M, Pagliani T, Parini P, and Moschetta A (2010) Down-regulation of the LXR transcriptome provides the requisite cholesterol levels to proliferating hepatocytes. Hepatology 51:1334-1344

Lu P, Yan J, Liu K, Garbacz WG, Wang P, Xu M, Ma X, and Xie W (2015) Activation of aryl hydrocarbon receptor dissociates fatty liver from insulin resistance by inducing fibroblast growth factor 21. Hepatology 61:1908-1919.

Mannery YO, Ziegler TR, Park Y, and Jones DP (2010) Acetaminophen elimination half-life in humans is unaffected by short-term consumption of sulfur amino acidfree diet. J Pharmacol Exp Ther 333:948-953.

Michaut A, Moreau C, Robin MA, and Fromenty B (2014) Acetaminopheninduced liver injury in obesity and nonalcoholic fatty liver disease. Liver Int 34: e171-e179.

Miyakawa K, Albee R, Letzig LG, Lehner AF, Scott MA, Buchweitz JP, James LP, Ganey PE, and Roth RA (2015) A cytochrome P450-independent mechanism of acetaminophen-induced injury in cultured mouse hepatocytes. J Pharmacol Exp Ther 354:230-237.

Nam EJ, Hayashida K, Aquino RS, Couchman JR, Kozar RA, Liu J, and Park PW (2017) Syndecan-1 limits the progression of liver injury and promotes liver repair in acetaminophen-induced liver injury in mice. Hepatology 66:1601-1615.

Potter WZ, Davis DC, Mitchell JR, Jollow DJ, Gillette JR, and Brodie BB (1973) Acetaminophen-induced hepatic necrosis. 3. Cytochrome P-450-mediated covalent binding in vitro. J Pharmacol Exp Ther 187:203-210.

Ren S and Ning Y (2014) Sulfation of 25-hydroxycholesterol regulates lipid metabolism, inflammatory responses, and cell proliferation. Am J Physiol Endocrinol Metab 306:E123-E130.

Rhee J, Inoue Y, Yoon JC, Puigserver P, Fan M, Gonzalez FJ, and Spiegelman BM (2003) Regulation of hepatic fasting response by PPARgamma coactivator-1alpha (PGC-1): requirement for hepatocyte nuclear factor 4alpha in gluconeogenesis. Proc Natl Acad Sci USA 100:4012-4017.

Saini SP, Zhang B, Niu Y, Jiang M, Gao J, Zhai Y, Hoon Lee J, Uppal H, Tian H, Tortorici MA, et al. (2011) Activation of liver X receptor increases acetaminophen clearance and prevents its toxicity in mice. Hepatology 54:2208-2217.
Seo YK, Mirkheshti N, Song CS, Kim S, Dodds S, Ahn SC, Christy B, Mendez-Meza R, Ittmann MM, Abboud-Werner S, et al. (2013) SULT2B1b sulfotransferase: induction by vitamin $\mathrm{D}$ receptor and reduced expression in prostate cancer. $\mathrm{Mol}$ Endocrinol 27:925-939.

Shi X, Cheng Q, Xu L, Yan J, Jiang M, He J, Xu M, Stefanovic-Racic M, Sipula I, O'Doherty RM, et al. (2014) Cholesterol sulfate and cholesterol sulfotransferase inhibit gluconeogenesis by targeting hepatocyte nuclear factor $4 \alpha$. Mol Cell Biol 34: 485-497.

Tirona RG, Lee W, Leake BF, Lan LB, Cline CB, Lamba V, Parviz F, Duncan SA, Inoue Y, Gonzalez FJ, et al. (2003) The orphan nuclear receptor HNF4alpha determines PXR- and CAR-mediated xenobiotic induction of CYP3A4. Nat Med $\mathbf{9}$ : $220-224$.

Vallianou I, Dafou D, Vassilaki N, Mavromara P, and Hadzopoulou-Cladaras M (2016) Hepatitis C virus suppresses hepatocyte nuclear factor 4 alpha, a key regulator of hepatocellular carcinoma. Int J Biochem Cell Biol 78:315-326.

Vickman RE, Crist SA, Kerian K, Eberlin L, Cooks RG, Burcham GN, Buhman KK $\mathrm{Hu} \mathrm{CD}$, Mesecar AD, Cheng L, et al. (2016) Cholesterol sulfonation enzyme, SULT2B1b, modulates AR and cell growth properties in prostate cancer. Mol Cancer Res 14:776-786.

Villablanca EJ, Raccosta L, Zhou D, Fontana R, Maggioni D, Negro A, Sanvito F, Ponzoni M, Valentinis B, Bregni M, et al. (2010) Tumor-mediated liver X receptoralpha activation inhibits CC chemokine receptor-7 expression on dendritic cells and dampens antitumor responses. Nat Med 16:98-105.

Wang F, Beck-García K, Zorzin C, Schamel WW, and Davis MM (2016) Inhibition of $\mathrm{T}$ cell receptor signaling by cholesterol sulfate, a naturally occurring derivative of membrane cholesterol. Nat Immunol 17:844-850.

Wang Z, Yang X, Chen L, Zhi X, Lu H, Ning Y, Yeong J, Chen S, Yin L, Wang X, et al. (2017) Upregulation of hydroxysteroid sulfotransferase $2 \mathrm{~B} 1 \mathrm{~b}$ promotes hepatic oval cell proliferation by modulating oxysterol-induced LXR activation in a mouse model of liver injury. Arch Toxicol 91:271-287.

Wolf SJ, Heard K, Sloan EP, and Jagoda AS; American College of Emergency Physicians (2007) Clinical policy: critical issues in the management of patients presenting to the emergency department with acetaminophen overdose. Ann Emerg Med 50:292-313.

Xu J, Xu Y, Li Y, Jadhav K, You M, Yin L, and Zhang Y (2016) Carboxylesterase 1 is regulated by hepatocyte nuclear factor $4 \alpha$ and protects against alcohol- and MCD diet-induced liver injury. Sci Rep 6:24277.

Yamamoto A, Liu MY, Kurogi K, Sakakibara Y, Saeki Y, Suiko M, and Liu MC (2015) Sulphation of acetaminophen by the human cytosolic sulfotransferases: a systematic analysis. J Biochem 158:497-504.

Yang X, Xu Y, Guo F, Ning Y, Zhi X, Yin L, and Li X (2013) Hydroxysteroid sulfotransferase SULT2B1b promotes hepatocellular carcinoma cells proliferation in vitro and in vivo. PLoS One 8:e60853.

Yin L, Ma H, Ge X, Edwards PA, and Zhang Y (2011) Hepatic hepatocyte nuclear factor $4 \alpha$ is essential for maintaining triglyceride and cholesterol homeostasis. Arterioscler Thromb Vasc Biol 31:328-336.

Yoon E, Babar A, Choudhary M, Kutner M, and Pyrsopoulos N (2016) Acetaminopheninduced hepatotoxicity: a comprehensive update. J Clin Transl Hepatol 4:131-142.

Zhang J, Huang W, Chua SS, Wei P, and Moore DD (2002) Modulation of acetaminopheninduced hepatotoxicity by the xenobiotic receptor CAR. Science 298:422-424.

Address correspondence to: Wen Xie, Department of Pharmaceutical Sciences, University of Pittsburgh, 306 Salk Pavilion, Pittsburgh, PA 15261. E-mail: wex6@pitt.edu 